\title{
From the Valley of Death to the Crossroads of Opportunity: A Discussion of Evolving Benefit/Risk Evaluation Standards
}

Therapeutic Innovation \& Regulatory Science 2018, Vol. 52(5) 53I-536 (C) DIA 2018 Article reuse guidelines: sagepub.com/journals-permissions DOI: $10.1177 / 2168479018774556$ tirs.sagepub.com

\author{
Peter J. Pitts, BA ${ }^{1,2}{ }^{\infty}$, and Patrick Brady, PharmD ${ }^{3}$
}

\begin{abstract}
A series of recent US Food and Drug Administration (FDA) approvals (such as Sarepta's Exondys 5 I, Merck's Keytruda, and Portola's Bevyxxa) has generated significant interest within the drug development ecosystem. Facilitated regulatory pathways aimed toward expediting medicines to patients suffering from serious and life-threatening conditions are a good thing, even if it raises curiosity and introduces some degree of uncertainty. Over the last 20 years, two key words in drug development have been speed and innovation. Going forward, the patient voice, data quality, and evidence generation must be added to that list. There is a raging debate over the level of evidence expected to first introduce a treatment to patients. Some argue for less data followed by postapproval follow-up, others for more adaptive clinical trial designs and end-point modification driven by patient-focused drug development and use of real-world evidence. The transition in the regulatory framework is happening in front of our eyes. How are these shifts in regulatory science interpreted within the context of 2 Ist-century drug development-and how can these learnings help advance patient care while placing into context the expected uncertainty we find in benefit-risk data?
\end{abstract}

\section{Keywords}

expedited review pathways, risk-benefit analysis, adaptive clinical trials, real world evidence, patient-focused drug development

A series of recent US Food and Drug Administration (FDA) approvals (such as Sarepta's Exondys 51 $1^{\mathrm{i}}$, Merck's Keytrudai, and Portola's Bevyxxaiii) has generated significant interest within the drug development ecosystem. Facilitated regulatory pathways aimed toward expediting medicines to patients suffering from serious and life-threatening conditions are a good thing, even if it raises curiosity and introduces some degree of uncertainty. Over the last 20 years, 2 key words in drug development have been speed and innovation. Going forward, the patient voice, data quality, and evidence generation must be added to that list. There is a raging debate over the level of evidence expected to first introduce a treatment to patients. Some argue for less data followed by post-approval follow-up, others for more adaptive clinical trial designs and end-point modification driven by patient-focused drug development and use of real-world evidence. The transition in the regulatory framework is happening in front of our eyes. How are these shifts in regulatory science interpreted within the context of 21st-century drug development - and how can these learnings help advance patient care while placing into context the expected uncertainty we find in benefit-risk data?

\section{I st-Century Regulatory Dimensionality: The Power of the Patient Voice}

The approval of Exondys 51 (for Duchenne muscular dystrophy) struck several chords both inside and outside the walls of
FDA headquarters in White Oak, MD. According to then FDA commissioner Robert Califf, "The science is not in dispute beyond the usual types of disagreement that occur when experts review clinical evidence from different perspectives.... It is clear that Dr. Woodcock's decision utilized the flexibility afforded under the relevant statutory provisions, including consideration of the life-threatening decisions of the disease and the lack of alternative treatments. "iv The pharmaceutical industry is no stranger to stakeholders rendering different perspectives over the same data, and this will become increasingly so as patients and payers grow their influence in decision making.

It is noteworthy that Dr Janet Woodcock, Director of the FDA's Center for Drug Evaluation and Research (CDER), offered these comments at Exondys 51's Peripheral and Central Nervous System Drugs Advisory Committee meeting, "It's

\footnotetext{
' Center for Medicine in the Public Interest, New York, NY, USA

${ }^{2}$ École Supérieure des Sciences Économiques et Commerciales (Paris/Singapore), New York, NY, USA

${ }^{3}$ Bayer Corporation, Whippany, NJ, USA
}

Submitted 7-Apr-2018; accepted I2-Apr-2018

Corresponding Author:

Peter J. Pitts, Center for Medicine in the Pubic Interest, 757 Third Avenue, 20th Floor, New York, NY 10017, USA.

Email: ppitts@cmpi.org 
possible to reach different conclusions based on the data presented today.... Failing to approve a drug that actually works in devastating diseases - these consequences are extreme."”

Exondys 51 was approved under the accelerated approval pathway, which provides for the approval of drugs that treat serious or life-threatening diseases and generally provide a meaningful advantage over existing treatments. Approval under this pathway can be based on adequate and well-controlled studies showing the drug has an effect on a surrogate endpoint that is reasonably likely to predict clinical benefit to patients (eg, how a patient feels or functions or whether they survive).

The FDA also granted Exondys 51 fast track designation, which is a designation to facilitate the development and expedite the review of drugs that are intended to treat serious conditions and that demonstrate the potential to address an unmet medical need. It was also granted priority review and orphan drug designation. Priority review status is granted to applications for drugs that, if approved, would be a significant improvement in safety or effectiveness in the treatment of a serious condition. Orphan drug designation (fewer than 200,000 patients) provides incentives such as clinical trial tax credits, user fee waiver, and eligibility for orphan drug exclusivity to assist and encourage the development of drugs for rare diseases.

The uniqueness of the debate is that it is not entirely over the clinical trial design or robustness of the clinical data, or other traditional criteria-it's about "failing to approve a drug that actually works." Is this a new regulatory standard? What does it mean? How is it defined and measured? In the past, regulators have been accused of embracing ambiguity to allow it flexibility to deny approvals - can we now expect similar regulatory ambiguity that results in unanticipated approvals?

While there is increasing disagreement among stakeholders over the extent to which randomized clinical trial data should support the approval of a new medicine, the diversity of scientific viewpoints surrounding the data describing the benefits and risks of Exondys 51 underscores a wider debate about the pressures that decision makers face in balancing uncertainty against access for patients who desperately seek treatment. The 21 st-century patient voice represents the key variable in a new regulatory dimensionality.

In a recent podcast, former FDA Commissioner Dr. Califf commented that when there is a life-threatening disease with no effective treatment, "patient groups have been very clear that they are willing to take a high degree of risk to have earlier access., "vi

In this case, risk should be interpreted as uncertainty. A risk that is worthwhile when patients literally have no other option. A key benefit of PDUFA VI is to facilitate the systematic integration of the patient perspective into the development and approval process of new medicines. ${ }^{\text {vii }}$ Patients, payers, and drug developers should expect to see more public discussion and regulatory decision making that follow a similar path.

Even so, how does this compel industry to think differently about data?
As Dr Califf and Deputy Commissioner Rachel Sherman (now Principal Deputy Commissioner under Commissioner Scott Gottlieb) commented, "Creating knowledge requires the application of proven analytical methods and techniques to biomedical data in order to produce reliable conclusions... There must be a common approach to how data is presented, reported and analyzed and strict methods for ensuring patient privacy and data security.... Rules of engagement must be transparent and developed through a process that builds consensus across the relevant ecosystem and its stakeholders ... To ensure support across a diverse ecosystem that often includes competing priorities and incentives, the system's output must be intended for the public good and be readily accessible to all stakeholders.",viii

Yes, Serapta's clinical program was flawed. But there was undeniable evidence of ... hope.

Is "hope" now considered a validated biomarker? Certainly not-but the FDA's Patient-Focused Drug Development program has begun to shift the agency-advocate relationship from anecdote to ally. How much uncertainty is a patient willing to accept? How can that tolerance be measurably factored into an acceptable benefit-risk calculation?

The tool set for using a more data-driven, regulatory-savvy patient voice is nascent and the tasks are as daunting as the opportunities, but it's a valuable new opportunity to drive a more patient-centered focus on how we view and interpret data. Such a mindset opens up the opportunity to embrace a more complete life-cycle approach to the regulation of health care technologies. A more dispassionate understanding of patient engagement in regulatory decision making, combined with a more mature expression of how data evolve over time, upends the traditional frame of regulatory stasis and introduces the opportunity for a much more dynamic understanding of a medicines benefits and risks that extends far beyond what is understood at approval. And this is particularly relevant for those innovative medicines approved via new regulatory pathways.

\section{Beyond Speed: Understanding the Impact of Expedited Regulatory Pathways}

In a 2005 In Vivo article, then Acting CDER Director, Dr Steven Galson (now Senior Vice President for Global Regulatory Affairs at Amgen) commented that "just because a company has a huge vested interest in getting a product approved quickly, doesn't mean that it meets the very strict public health criteria for accelerated approval., ${ }^{\text {,ix }} \mathrm{A}$ lot of water has passed under the bridge and today there are many more options for swifter FDA review. In addition to Accelerated Approval there are review pathway designations such as Fast Track, Breakthrough Therapy, and Priority Review. ${ }^{x}$

In a Balancing the Need for Access With the Imperative for Empirical Evidence of Benefit and Risk, a recent editorial in the Journal of the American Medical Association (JAMA), ${ }^{\mathrm{xi}}$ Dr Califf writes that "substantial progress in balancing safety 
with access to effective therapies will come from systemic changes in the ecosystem rather than incremental modifications made by imposing more severe demands on individual products."

Again, there should be a distinction between safety and uncertainty. It is clear that patients are willing to tolerate varying degrees of risk in light of different degrees of benefit, but how much benefit is expected? Some uncertainty will always exist, but it is greatest at the time of review.

\section{Case in Point}

This evolving approach to data, uncertainty, and evidence generation is accessible in the agency's actions relative to the Merck product Keytruda. In May 2017, the FDA issued a press release announcing that they had "granted accelerated approval to a treatment for patients whose cancers have a specific genetic feature (biomarker). This is the first time the agency has approved a cancer treatment based on a common biomarker rather than the location in the body where the tumor originated."xii

"This is an important first for the cancer community," said Richard Pazdur, MD, acting director of the Office of Hematology and Oncology Products in the FDA's CDER and director of the FDA's Oncology Center of Excellence. "Until now, the FDA has approved cancer treatments based on where in the body the cancer started - for example, lung or breast cancers. We have now approved a drug based on a tumor's biomarker without regard to the tumor's original location."xiii

Within Merck, the Keytruda strategy inspired a shakeup of Merck's historically conservative and linear drug development mindset and reassured Merck management that its new approach-involving doing more work at risk, and with far greater agility - was regarded favorably by regulators and represented a move in the right direction. ${ }^{\text {xiv }}$

A win for the FDA. A win for patients. A win for Merck. But what is not so readily visible are the difficult and risky decisions required by both agency (benefit-risk balance) and developer (investment, commercial viability). In addition, there is the ongoing debate over the regulatory validity and/or the commercial viability of new medicines approved via biomarkers. As a Forbes "tick-tock" story on the Keytruda approval points out, "In general, commercial teams tend not to favor biomarkers and seek to avoid them wherever possible. They would rather target an entire population than a subpopulation, and also recognize the inconvenience of requiring a patient to get tested prior to using a drug." ${ }^{\mathrm{xv}}$

The larger problem, however, is existing uncertainty relative to what biomarkers can be used for regulatory review-and under what circumstances? To overcome this lack of regulatory predictability requires a greater degree of acceptance toward the uncertainty of biomarkers themselves by both drug developers and regulators. In this respect, the Keytruda story is more of a positive anecdote than a predictive data point in plotting future agency decision-making variables.
Biomarkers can help to measure uncertainty on an important parameter that can be demonstrated in a relatively short period of time, and this is important to patients. But, a data uncertainty plan should extend far beyond the time of approval.

\section{The Value Proposition of Adaptive Clinical Trials}

The FDA's February 2010 draft guidance on Adaptive Design Clinical Trials for Drugs and Biologics ${ }^{\mathrm{xvi}}$ was considered a watershed moment in 21 st-century regulatory science since moving even a few steps from the traditional randomized clinical trial (RCT) gold standard is still viewed by many as regulatory apostasy. Is the risk worth the benefit?

According to Steven Schwager, professor emeritus of statistics and biological statistics at Cornell University, a major disadvantage to adaptive clinical trials is the additional complexity in having to determine whether to make a change, when to make it and what kind of change to make. There are lots of ways to modify a trial, and it's possible, if not done carefully, for changes to make the design worse rather than better. ${ }^{\text {vvii }}$

This can also lead to operational challenges in the clinical supply chain. The supply chain needs to be much more nimble and flexible, because changes in the study dictate changes in the supply chain, data-monitoring committees need to be able to review the data, and it can be a challenge to extract and analyze it, derive what changes are needed, and express them in a way that drug-monitoring committees can digest and use to make quick decisions.

Theory met reality in the FDA's review of Pertola's Bevyxxa. According to the FDA's June 23, 2017, press release,

Approval was based on data from APEX (NCT01583218), a randomized, double-blind, multinational clinical trial comparing extended duration Bevyxxa (35 to 42 days) to short duration of enoxaparin ( 6 to 14 days) in the prevention of VTE [venous thromboembolism] in an acutely medically ill hospitalized population with risk factors for VTE. The trial randomized 7,513 patients to either Bevyxxa or enoxaparin treatment. Patients on the Bevyxxa arm took an initial dose of $160 \mathrm{mg}$ orally on day 1 , then took $80 \mathrm{mg}$ once daily for 35 to 42 days and received a placebo injection once daily for 6 to 14 days. Patients on the enoxaparin arm received 40 mg subcutaneously once daily for 6 to 14 days and took a placebo pill orally once daily for 35 to 42 days.

Efficacy was measured in 7,441 patients by a composite outcome score comprised of either the occurrence of asymptomatic or symptomatic proximal deep vein thrombosis, non-fatal pulmonary embolism, or VTE-related death. Fewer events were observed in patients receiving Bevyxxa (4.4\%) compared with those taking enoxaparin (6\%) (relative risk $0.75,95 \%$ CI: $0.61,0.91){ }^{\text {xviii }}$

The adaptive design is important because the approval of Bevyxxa was based on reanalysis of data from a randomized, double blind, double-dummy trial that compared extendedduration Bevyxxa to short-duration enoxaparin in acutely medically ill hospitalized subjects at risk for VTE. ${ }^{\text {xix }}$ 
A total of 7,441 subjects met the criteria of efficacy analyses (3,721 for Bevyxxa, and 3,720 for enoxaparin). The primary outcome occurred in 165 subjects who received Bevyxxa $(4.4 \%)$ and $223(6.0 \%)$ in the enoxaparin arm (25\% risk reduction). Subjects randomized to the reduced dose showed no difference in efficacy outcome ( $6.9 \%$ vs. $6.7 \%$, respectively) and corresponding $42 \%$ lower median drug plasma levels. Results from a retrospective substudy suggest Bevyxxa also reduced all-cause stroke and ischemic stroke compared to enoxaparin through 77 days of follow-up. ${ }^{\mathrm{xx}}$

There haven't been any substantial arguments against the validity of either the adaptive nature of the trial design or the statistical validity of the data; nevertheless there remains a fundamental (and, in many ways existential) question - at what point does an agency review end and, once an approval is given, how does labeling present the evidence for benefit/risk in specific patient (sub)populations? These are on-going regulatory questions and, as with the "advancing regulatory science" issues discussed above concerning biomarker validation (or prevalidation), there's good news (more therapeutic options) and not so good news (lack of predictive agency rules, guidance, or just ... behavior).

\section{Too Fast or Too Slow: Is the FDA Moving Forward or Backward?}

When it comes to regulatory science and the broader issues facing the FDA, there will always be significant gradations of nuance and ambiguity. "Predictability" is a trail that regulator and regulated must blaze together, sometimes heroically and at other times with greater caution.

Is the FDA approving drugs too fast or not fast enough? Are they demanding too much data or not enough? There isn't any dearth of commentary supporting either proposition. There is, however, no evidence to support the sound byte that the FDA is approving "everything," or that every product that requests an expedited pathway receives it, or that "all" those that do receive an expedited pathway designation get approved, or that every product that does reach the market via an expedited approval is in some way more dangerous than other medicines. In shortdon't believe the hype. Some particulars:

- An analysis of every product (364) requesting a Breakthrough Therapy designation (from July 2012-June 2016) shows that CDER granted $133(37 \%)$ of those requests, denied $182(50 \%)$, and the sponsor withdrew their request 49 times (13\%) before the agency made a decision. ${ }^{\text {xxi }}$ Hardly a regulatory carte blanche.

- In 2013, the first full year of the Breakthrough Designation, the FDA approved 3 new drugs, 14 in 2014, and 9 in 2015. ${ }^{\text {xxii }}$ Hardly an onslaught of new medicines.

- Among 22 drugs with 24 indications granted accelerated approval by the FDA in 2009-2013, efficacy was often confirmed in post-approval trials a minimum of 3 years after approval, although confirmatory trials and preapproval trials had similar design elements, including reliance on surrogate measures as outcomes. ${ }^{\text {xxii }}$

Unsafe? Not effective? "Dangerous"? A new "wild west" FDA? No.

Policies and regulations that seek to limit risk are often shaped by the immediate fear of sensational events. This perspective is commonly called "The Precautionary Principle," which in various forms asserts that unless innovators can demonstrate that a new technology is risk free, it should be not allowed into the marketplace. Moreover, any product that could possibly be dangerous at any level should be strictly and severely regulated. In reality, this punishes patients and stymies innovation. There is never a medicine that is $100 \%$ safe, and there is always some degree of uncertainty that exists.

Joshua Lederberg, the Nobel Prize Laureate, once observed that the failure of regulatory, legal, and political institutions to integrate scientific advances into risk selection and assessment was the most important barrier to improved public health.

According to Lederberg, "the precedents affecting the longterm rationale of social policy will be set, not on the basis of well-debated principles, but on the accidents of the first advertised examples." "xxiv

One aspect of expedited review protocols that is often overlooked or ignored entirely is the participation of senior agency resources outside of the particular responsible division (both within and outside of CDER's Medical Policy Council ${ }^{\mathrm{xx}}$ ). This means that not only can difficult questions be addressed more speedily but potentially more advanced "regulatory discretion" can be used to overcome potential deal-breaker issues.

New science and the strategies and tactics to incorporate them into regulatory thinking does not mean a free pass for bad science. What it does mean is that the FDA (and from the highest levels) is rightly embracing regulatory dimensionality, a combination of scrupulous review processes and pragmatism. Together with a recalibrated sense of regulatory velocity (Speed + Accuracy + Public Health Need), it's the agency's next step toward a more entrepreneurial regulatory attitude. The FDA as innovation accelerator.

What does this mean for drug developers and their regulatory strategists? Does it somehow minimize the importance of traditional success metrics such as first-cycle approvals? Certainly not. What it does mean is, as more and more complex molecules are investigated, different regulatory approaches need to be discussed, debated, and considered. How can "the patient voice" be incorporated into both clinical and regulatory discussions? What are the public health imperatives that might appropriately drive the FDA to positively view a request for one or more expedited pathways? Importantly, it's critical to understand that a truncated clinical program and shorter review via an expedited review pathway does not mean a less rigorous FDA interrogation of the sponsor. It will mean a different approach with different roles and responsibilities on the part of both agency and sponsor. And it will almost certainly mean more time, effort, and expense in the postmarket environment. 


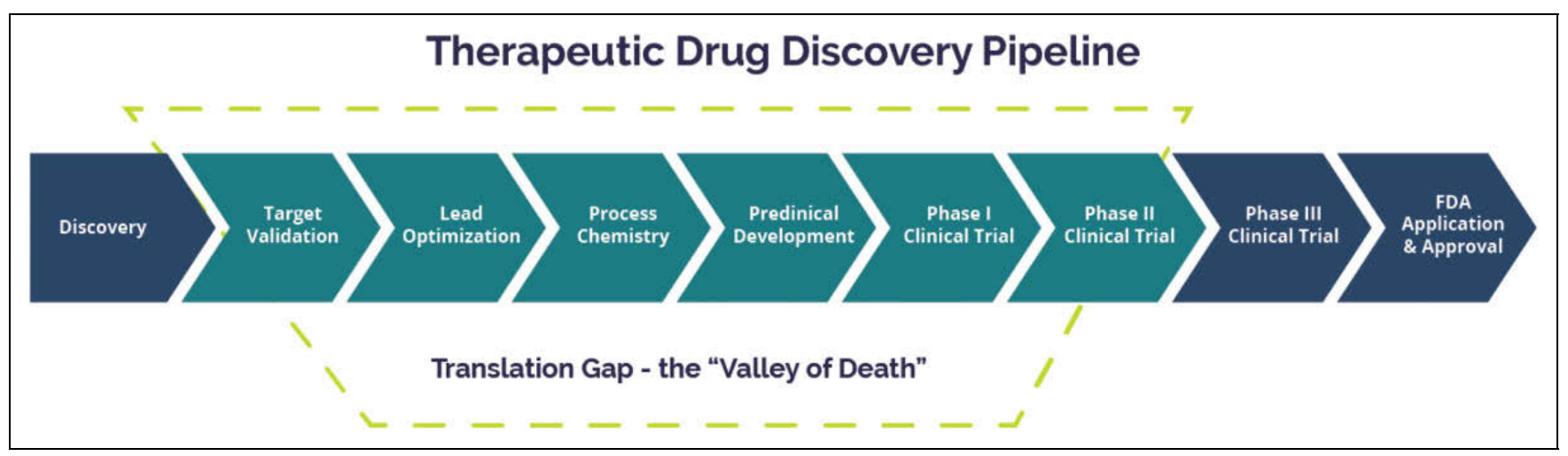

Figure I. Therapeutic drug discovery pipeline.

The Crossroads of Opportunities also offers industry regulatory strategists the opportunity to help maximize broader corporate investment. The heretofore-dreaded "Valley of Death" (see Figure 1) can be more aggressively and accurately navigated through more creative internal partnerships earlier in the development process.

New regulatory opportunities, creatively considered and appropriately executed, can help developers better navigate the "Valley of Death" and avoid a "Valley of Debt."

Doing things in new ways with new tools is always challenging. Drug developers and their regulatory strategists are at a crossroads. The 21 st century regulatory strategist must question and consider new possibilities to achieve what's right for the company, what's right for the molecule as well as what's right for the public health - because those are the same questions and considerations being asked and provided by the $21 \mathrm{st}$ century Food and Drug Administration.

It is not necessary to change.... Survival is not mandatory.

-W. Edwards Deming

\section{Acknowledgment}

The authors would like to acknowledge the important contributions of Joseph Scheeren, PharmD, Senior Advisor, Research and Development, Bayer; Todd Paporello, PharmD, MBA, Vice President and Head of North American Regulatory Affairs Pharma and Consumer Health, Bayer; and Kim Quaintance-Lunn, Vice President, Regulatory Policy, North America, Bayer. Their assistance in reviewing this paper throughout its development has resulted in a far superior presentation than the authors alone could have provided.

\section{Declaration of Conflicting Interests}

Mr Pitts has completed several consulting projects for Bayer Healthcare Pharmaceuticals.

\section{Funding}

No financial support of the research, authorship, and/or publication of this article was declared.

\section{ORCID iD}

Peter J. Pitts D http://orcid.org/0000-0003-1445-927X

\section{Notes}

i. https://www.fda.gov/newsevents/newsroom/pressannounce ments/ucm521263.htm.

ii. https:/www.accessdata.fda.gov/drugsatfda_docs/appletter/ 2016/125514orig1s009ltr.pdf.

iii. https://www.fda.gov/drugs/informationondrugs/approved drugs/ucm564422.htm.

iv. https://www.gpo.gov/fdsys/pkg/PLAW-112publ144/pdf/ PLAW-112publ144.pdf.

v. https://www.statnews.com/pharmalot/2016/04/25/fda-panelsarepta-muscular-dystrophy/.

vi. https://jamanetwork.com/learning/audio-player/14650592.

vii. https://phrma.org/sites/default/files/pdf/Factsheet-PDUFAVI.pdf.

viii. http://blogs.fda.gov/fdavoice/index.php/2016/05/what-wemeanwhen-we-talk-about-evgen-part-ii-building-out-anational-systemfor-evidence-generation/.

ix. Hurry up and wait: the slowdown in accelerated approvals, In Vivo Magazine, July/August 2005.

x. https://www.focr.org/fda-expedited-review-programs.

xi. http://jamanetwork.com/journals/jama/article-abstract/ 2648611.

xii. https:/www.fda.gov/newsevents/newsroom/pressannounce ments/ucm560167.htm.

xiii. ibid.

xiv. https://www.forbes.com/sites/davidshaywitz/2017/07/26/thestartling-history-behind-mercks-new-cancer-blockbuster/4/ \#10e44d957b18.

xv. https:/www.forbes.com/sites/davidshaywitz/2017/07/26/thestartling-history-behind-mercks-new-cancer-blockbuster/4/ \#6c0866047b18.

xvi. https://www.fda.gov/downloads/Drugs/.../Guidances/ ucm201790.pdf.

xvii. https:/www.forbes.com/sites/medidata/2016/03/16/when-isadaptive-design-right-for-your-clinical-trial/\#2dd8e7052e55. 
xviii. https://www.fda.gov/drugs/informationondrugs/approved drugs/ucm564422.htm.

xix. Bevyxxa Prescribing Information. Portola Pharmaceuticals, Inc. June 2017.

xx. https://www.ahcmedia.com/articles/141285-betrixaban-cap sules-bevyxxa.

xxi. http://journals.sagepub.com/doi/full/10.1177/2168479017 696284.

xxii. https://www.fda.gov/downloads/Drugs/DevelopmentApproval Process/SmallBusinessAssistance/UCM466480.pdf.

xxiii. http://jamanetwork.com/journals/jama/article-abstract/ 2648631. xxiv. https://books.google.com/books?id=SggAAAAAMBAJ\& $\mathrm{pg}=\mathrm{PA} 4 \& \mathrm{lpg}=\mathrm{PA} 4 \& \mathrm{dq}=\mathrm{joshua}+$ lederberg + the + precedents affecting + the + long-term + rationale + of + social + policy + will + be + set,+ not + on + the + basis + of + well-debated + principles, + but + on + the + accidents + of + the + first + advertised + examples \& source $=$ bl\&ots $=$ zdOUmdwoVv\&sig $=q 9 z T F O \_u R 8 F j W Q$ FuPVNndq1ZV4o\& $\mathrm{hl}=$ en $\&$ sa $=\mathrm{X} \& \mathrm{ved}=0 \mathrm{ahUKEwjy} 48 \mathrm{KQ}$ 6oHWAhXDRiYKHSCrCOcQ6AEILjAC\#v=onepage\&q\& $\mathrm{f}=$ false.

xxv. https:/www.fda.gov/downloads/AboutFDA/CentersOffices/ OfficeofMedicalProductsandTobacco/CDER/ManualofPoli ciesProcedures/UCM344444.pdf 